\title{
Covid-19 Remote consultations for physician associates in primary care: qualitative feedback from a regional cohort study
}

\author{
Authors: Julie Hoskin ${ }^{A}$ and Ria Agarwal ${ }^{B}$
}

The advent of the COVID-19 pandemic necessitated a dramatic shift in the traditional general practice (GP) model of consultation. General practice was forced to deliver care remotely and this workforce included physician associates (PAs), who may not have been previously exposed to remote consultations prior to the pandemic. A qualitative online questionnaire explored how a cohort of primary care PAs in Sheffield adapted to the use of remote consultations, how clinically safe they felt in utilising this method, supervision arrangements in their practices and how they would manage three clinical scenarios. Recommendations are limited by the small sample size, but based upon this feedback we recommend inclusion of remote consultation as part of the student experience in higher educational institutions (HEIs) that do not currently utilise it; discussion of the PAs' previous experience of remote consultation at their induction in order to decide how to most effectively use their skillset while they transition into remote consultations, appreciating that they may have a lower threshold to invite patients in for a face-to-face appointment if newly qualified; on-demand supervision for remote consultations where possible; and reinforcement of clinical and pastoral review from the employer to manage the increasing scope of the PA.

KEYWORDS: physician associate, remote consultations, primary care, preceptorship scheme, telephone consultation

DOI: $10.7861 /$ fhj.2021-0023

Authors: ${ }^{A}$ advanced nurse practitioner and physician associate preceptorship lead, Sheffield Clinical Commissioning Group, Sheffield, UK; ${ }^{\text {B }}$ physician associate, Sothall Medical Centre, Sheffield, UK, senior lecturer, Sheffield Hallam University, Sheffield, UK and allied health professional representative, Royal College of General Practitioners South Yorkshire, Sheffield, UK

\section{Physician associate training}

Higher education institutions (HEIs) follow a central curriculum based upon the matrix specification of core clinical conditions for the physician assistant and the competence and core curriculum framework for the physician assistant. ${ }^{1,2}$ The General Medical Council (GMC) announced an agreement to regulate the physician associate (PA) profession in July 2019, and this process will include quality assurance of HEIs delivering PA courses. ${ }^{3}$ While this process is pending, there is likely to be some level of variation between HEIs in regards to exact delivery of their PA course. A PA course is also extremely intensive, as their 2-year training is based upon the medical model and encompasses clinically focused theoretical learning and applied learning through placement exposure. On completion of the course, they sit a national exam, which then allows them to practise as a PA on the Physician Associate Managed Voluntary Register.

\section{Physician associates working in Sheffield}

There is an established PA preceptorship scheme that has been running in Sheffield since the city's first PAs graduated in 2018, supported financially and with staffing by the NHS Sheffield Clinical Commissioning Group (CCG). ${ }^{4}$ Graduates from the two local HEI's contribute to the PA preceptorship scheme numbers, but the scheme has also attracted a minority of PAs who trained outside of Sheffield. This scheme enables practices to recruit PAs as sole employers rather than as a primary care network (PCN), and the growth of this scheme has since allowed 16 (out of the 78 practices across the city) to benefit from this initiative. Several practices within the scheme have appointed two PAs following successful integration of the first PA and, in one case, four PAs. Regular contact with PAs previously or currently employed as part of this scheme built a picture to the authors of an overnight transition into remote consultation for the majority of these PAs; many of whom informally stated that they'd had little formal training or practice in remote consultations prior to the pandemic. The Interim NHS People Plan estimates that there will be 5,900 PA graduates by the end of $2023 .{ }^{5}$ Consequently, the authors felt a need to ensure that these new members of the workforce are working safely and effectively despite the COVID-19 pandemic. While this cohort is small, it has been selected purposefully to 
manage regional differences between HEI's delivering PA courses, and we hope that our findings will be of interest to HEIs and primary care employers.

\section{Definition of / background to the 'remote consultation'}

In order to reflect current practice in primary care, we define a remote consultation as a method of consulting where the patient is not physically present in the room with the clinician, but a similar amount of information is gathered through telephone or video formats to guide diagnosis and management. ${ }^{6}$ This remains separate from what we would define as a triage, in which a shorter amount of information is gathered by a clinician to decide the next steps.

Anecdotally, telephone consultations and triage were often undertaken by more experienced general practitioners (GPs), who had the benefit of experience and knowing their patient population to make clinical decisions over the phone. While remote consultation has much to recommend it, the way in which it has occurred due to the pandemic left little time for a considered change in the education of those clinicians preparing to enter primary care.

One of the authors is a practising primary care PA of nearly 8 years and noted the overnight move into remote consultations was challenging for both her and the preceptorship PA at her surgery; both having only assisted with the duty doctor's telephone triage list on an ad hoc basis prior to the pandemic. Dealing with a higher degree of uncertainty over the telephone in addition to minimising face-to-face contact led to a discussion between the authors around how the newer workforce (such as the local PA community) were adapting. To try and probe this further, one of the authors delivered a case-based session to the preceptorship group in June 2020 on remote consultations, which suggested that the more experienced PAs within the group were better able to manage patient cases over a telephone consultation in comparison with those more newly qualified. In addition to more formally ensuring individuals within the group felt supported and clinically safe, the authors wanted to investigate approximately how much primary care experience a PA needed to feel comfortable in managing a patient remotely.

\section{Methodology}

A qualitative online questionnaire was sent in Summer 2020 to 22 primary care PAs working in the Sheffield and Rotherham areas (supplementary material S1). The questionnaire invited free-text responses to gauge a starting point among the group and enable inclusion of additional themes that are discussed later. We received 13 anonymised responses from PAs, which was lower than hoped, but likely due to the increased workload during the COVID-19 pandemic. This cohort had varying levels of clinical experience, ranging from less than 3 months to just over 3 years. One additional PA with 3 years of experience was not able to complete the survey but shared some informal feedback with the authors regarding their views on face-to-face appointment usage, which is also highlighted in the results section. While this cohort has limitations in sample size and representation, by limiting the survey to this group, we have ensured some consistency in external variables. All PAs were either currently or historically a formal recipient of the preceptorship scheme or voluntarily participated in its support streams (such as the weekly teaching sessions and communal group chat). These results are also limited to the responses written by the PA participants as opposed to a verbal response in a focus group or other more rigorous qualitative methodology. The authors felt the responses given were sufficient in quickly examining our hypothesis that newly qualified PAs may be less able to manage patients remotely.

\section{Results}

Four PAs had qualified since the onset of the COVID-19 pandemic, but due to the postponement of the national exams from May to September 2020, three of these had their student experience prior to the onset of the pandemic. All PAs who completed the survey performed some form of remote consultation, further details later. Due to the postponement of PA national exams with the onset of COVID-19, there were no PAs with 3-6 months experience, but otherwise there was fairly equal representation across the sample: four with 0-3 months' experience, four with 6-12 months' experience and five with $1+$ years' experience (Fig 1).

\section{Preparation for remote consultations}

Out of 13 responses, only one PA had primary care remote consultation experience. This was due to them starting their preceptorship at the onset of the pandemic. Consequently, they were able to opportunistically observe GPs doing remote consultation as part of their induction for 1 week. In the remaining 12 responses, two had practical experience of performing remote consultations prior to the pandemic (one for many years in their work as a psychologist prior to their PA training and the other during their student placement during the pandemic). They felt that prior experience of speaking with patients on the phone was helpful for them when starting work in this environment. Another two PAs with 1-3 years' experience had prior involvement in telephone triage but not remote consultation. A further two PAs reported observation only of remote consultations and/or telephone triage on student placements. One stated: 'I think I observed a GP doing triage for maybe a couple of hours at the beginning of the first primary care placement early in the course. It was so little and so long ago that I'm unsure how useful it was.'

Following the onset of the pandemic, two PAs were given some form of remote consultation training. For one, this consisted of a handout from an established GP continuing professional development company and, for the other, a two-hour online module, but both felt these were less meaningful than the actual experience of doing a remote consultation. The remaining PAs were not given any specific guidance, with one stating: 'We were

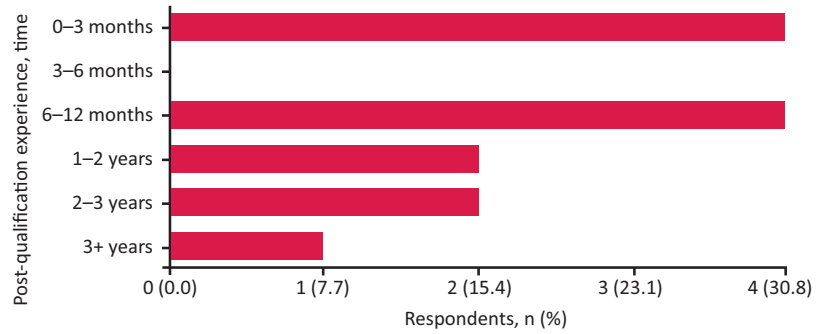

Fig 1. Length of primary care experience from physician associate respondents, $n=13$. 
expected to get on with it.' None of the cohort felt prepared to do remote consultations initially, but all stated they felt more able to do remote consultation with experience of this; one PA with 0-3 months' experience describing that it took a month for them to feel comfortable in remote consultation, highlighting a 'steep learning curve'.

\section{Types of consultation}

All respondents estimated having $60 \%-80 \%$ of their clinic as remote consultation appointments, with a subset of face-to-face appointments following this; bookable either by colleagues or by themselves. All bar one PA undertook remote consultations on complaints that they would usually have assessed face-to-face, although some discussed being able to select from a communal list. The remaining PA had $0-3$ months' experience, stating: 'I don't really do on the day appointments for patients. I see patients face-to-face for learning disability and severe mental illness reviews then do long-term condition reviews over the phone ... the doctors mainly do on the day telephone calls ... it is very different to what I imagined doing [as a PA student].'

In regards to the other PAs with 0-3 months' experience, the second described that any face-to-face appointments that they booked had to be discussed with a supervisor first, deeming this helpful for their learning. The third PA with 0-3 months' experience described sitting with their supervisor for a week sharing telephone calls together and thus observing each other to aid mutual confidence building. They described remote consultation as being anxiety-provoking due to the absence of usual cues, although they were getting used to it with clinical experience accruing. The final PA in this subset described just doing mental health and dermatology patients as remote consultations, but broader use of their face-to-face appointments pre-booked by others.

\section{Supervision}

All surveyed PAs felt supported by their supervision arrangement, which was fortunately available on demand for all PAs. A PA with 0-3 months' experience went on to say: 'I feel safe because I know I can talk to someone whenever I need to. Over the phone you can search something online quickly, message someone on the computer or ask the patient if you can call them back.'

\section{Face-to-face consultations}

One PA with 0-3 months' experience felt unhappy seeing patients booked by other clinicians and felt more prepared speaking to the patient first directly, but other PAs were happy that face-to-face appointments booked by other clinicians were appropriate; some PAs even stating that they appreciated the exposure for their examination skills. Another PA with 0-3 months' experience felt face-to-face appointments booked by other clinicians overran as they felt the need to retake the history again. All PAs who saw their own patients following their own remote consultation reported feeling satisfied with this process. Three PAs felt they were seeing face-to-face appointments booked in by other clinicians which could have been dealt with remotely, with one being unhappy that they had more face-to-face appointments than other clinicians. Another stated some of these patients were not appropriately screened for COVID-19 and at times felt at risk.

\section{Responses to the clinical scenarios}

Scenario 1: A 50-year-old patient with asthma and a 3-day history of productive cough

The PAs with 0-3 months' experience suggested bringing in the respiratory patient for a face-to-face COVID-19 assessment appointment (colloquially known as 'hot hub assessment') or alluded to not feeling totally comfortable managing this scenario remotely. Fuller histories or video consultations were also suggested by this cohort and some PAs with 6-12 months' experience suggested the use of investigations (such as sputum culture and peak flow). Despite this, those with more than 6 months experience were otherwise happy to manage the patient remotely with antibiotics $+/$ - oral steroids.

\section{Scenario 2: A 60-year-old woman with a 5-day history of dysuria and suprapubic pain}

All but one of the PAs with 0-3 months' experience and half of the PAs with 6-12 months' experience wanted a urinalysis first before treating, with another PA with 0-3 months' experience requesting a renal function check too. The remaining PAs felt comfortable with remote management via antibiotics, with all PAs with beyond 12 months' experience not requesting urine samples prior to treatment. Of note, in this example, the PA that had primary care placement experience during the pandemic did manage the patient in the same way as the 12 months + cohort.

\section{Scenario 3: A 30-year-old man with a 1-month history of feeling down and anxious}

All PAs were happy to manage this patient remotely with antidepressants and psychological therapy choices based upon the exact history. However, one PA with 0-3 months' experience suggested requesting blood tests prior to treatment, and another suggested they would see the patient in personal protective equipment (PPE) to encourage rapport building if this was requested by the patient. Other PAs suggested video consultation for this purpose.

\section{Discussion}

Based on the submissions from this cohort, PAs generally lacked experience of remote consultation prior to working in their practices, yet this cohort moved into this way of working at the onset of the COVID-19 pandemic. Based on the response, the feeling was that practical exposure to remote consultation was superior to any online learning or written advice that was provided. Three out of the four PAs with 0-3 months' experience were sheltered in varying degrees from 'on the day' remote consultations. Interestingly, this appears to be reinforced by the survey responses which demonstrated that the PAs with 0-3 months' experience did have a lower threshold for wanting face-to-face assessments or investigations than those with more primary care experience. Small sample size and the selection of cases provided limits for any generalisations that can be made, but our findings point to the fact that newly qualified PAs are likely to be inexperienced decision makers. Unnecessary face-toface consultations may also risk exposing patients, their families, clinicians and practice staff to harm.

There is a lack of definitive guidance with respect to what patient complaints are deemed unsuitable for remote consultation. ${ }^{7}$ This 
is likely to be the case for some time due to variation in clinician judgment and the complexity affecting such decision making. A range of factors were identified by Greenhalgh et al in use of remote consultation in primary care. ${ }^{8}$ These include but are not limited to patient group factors, the reason for consulting, the clinical relationship with the patient and practice ethos. ${ }^{8}$ It was interesting to note that most PAs were happy to manage the mental health scenario remotely, regardless of their experience level. This may be due to an increased number of clinical clues being available remotely, with the option to obtain further clues in a video consultation where required. Given this data and the authors' experience, a stepped approach to telephone consultation for PAs with 0-3 months' experience seems an appropriate safeguard as their confidence builds, especially if they have minimal student experience of performing remote consultation. As remote consultation is likely to be a feature of general practice in the future, guidance on how to ensure students from all HEIs receive consistent exposure to this method would be welcomed.

Previous work has established that these new entrants to the primary care workforce have a great contribution to make in supporting access and fulfilling patient demand. ${ }^{9}$ We were delighted that all of the PAs who responded to the survey felt supported by their employers. The comment regarding ondemand supervision being helpful has been supported by more recent feedback from two PAs on the scheme who have since identified a lack of on-demand supervision making timekeeping difficult. Consequently, we would recommend on-demand supervision through a senior colleague at the practice, but we recognise this must be balanced against resource and time constraints of the employer.

It was concerning that some additional comments made in the survey suggested that a minority of this cohort's motivation to remain in general practice have been impacted by the change to remote consultation and/or the stress of the current environment, although none have suggested an alternative work environment/ role that they would pursue and, at the time of writing, all remain in primary care. Some of these views may have been impacted by general dissatisfaction (such as the example given of the PA with more face-to-face appointments than their colleagues) alongside other anecdotal issues (such as limitations in salary or clinical progression). ${ }^{10}$

\section{Recommendations for practice}

While not all PAs will work in primary care upon qualification, remote consultation is an increasing feature in some secondary care specialties, and a unique aspect of the PA role is their ability to move between primary and secondary care. There may be a transitional period in which PAs will need to gain exposure and confidence in remote consultations. Previous research on clinical supervision of primary care PAs recommended a formal induction period to enable the PA to become embedded in the practice's ethos. ${ }^{11}$ Following the onset of the pandemic, a further recommendation within this induction period would also be to ascertain how familiar the PA is with remote consultations, and discussion of strategies to support the PA with this, if required. Some strategies included by the practices employing PAs in this study were an observational period with a supervisor, shared telephone consultation lists allowing observation of the PA or a stepped approach to telephone consultation commencing with a few areas with which the PA felt confident and competent, and where more clinical clues were available. As clinical experience develops, the list of appropriate consultations should then expand where safe to do so, and this could be discussed at periodic supervisor reviews. During these reviews, the PA's clinical progression could also be explored; for example, would they be happier with more face-to-face appointments to increase their confidence in certain complaints, or would they prefer more autonomy to book their own face-to-face appointments. ${ }^{12}$

Periodic pastoral care in PA supervision is likely to be of utmost importance during the pandemic to ensure the safety and continued progression of this workforce member. Such mentoring should also be malleable to match the growing confidence of the PA and decisions should be collaborative. Adopting this approach not only allows workload to be adjusted appropriately but can help with early recognition of problems or issues that might affect the retention of PAs in primary care.

\section{Future work}

Where the PA has access to a preceptorship scheme, focused sessions may also help build confidence in this area, although differences in opinion are likely to reflect the approach of their employing practice. Further discussion within practice regarding agreement of clinical thresholds to bring patients in for face-toface assessment may be beneficial for all clinicians to engage in to ensure consistency at practice level.

Based upon our findings, we have recommended that trainees should be encouraged to embrace remote consultation in preparation for their future working environment. A larger study would be needed to make any formal recommendations around this so, at this stage, we simply wish to raise awareness of the realities of working as a PA in primary care. Where this is not already being utilised, HEIs could support this process through formally introducing the concept of remote consultation into curricula, shared remote consultations in placements and use of simulated patients within a remote classroom environment.

A limitation from this research is the absence of any PAs with 3-6 months' experience, which in this case has allowed us to clearly distinguish PAs with 0-3 months' experience from the PAs with more than 6 months' experience. It would be interesting to note how attitudes to remote consultation change throughout through the first year of practice in a larger group. A larger thematic analysis would have stronger validity in future work, but we feel these data provide a starting point in clinical literature around this topic.

\section{Supplementary material}

Additional supplementary material may be found in the online version of this article at www.rcpjournals.org/fhj: S1 - Questionnaire.

\section{References}

1 Department of Health. Matrix specification of core clinical conditions for the Physician Assistant by category of level of competence. DH, 2006. www.fparcp.co.uk/webapp/data/ media/58eb57fd01018_Matrix_of_core_clinical_conditions.pdf

2 Faculty of Physician Associates. Competence and curriculum framework for the physician assistant. FPA, 2012.

3 General Medical Council. Bringing physician associates and anaesthesia associates into regulation. GMC, 2020. https://www.gmc-uk. 
org/pa-and-aa-regulation-hub/map-regulation/what-will-regulationlook-like accessed on 22.03.21.

4 Hoskin J, Agarwal R. Preceptorship scheme for newly qualified physician associates working in general practice in Sheffield. Clin Med 2020;20:e255-9.

5 British Medical Association. Medical associate professions briefing. BMA, 2021. www.bma.org.uk/advice-and-support/nhs-deliveryand-workforce/workforce/medical-associate-professions-briefing

6 Power E, Bostock N. How a pandemic changed the face of general practice. GP. www.gponline.com/pandemic-changed-face-generalpractice/article/1703338 [Accessed 23 March2021].

7 NHS England, NHS Improvement. Clinical guide for the management of remote consultations and remote working in secondary care during the coronavirus pandemic. National Institute for Health and Care Excellence, 2020. www.nice.org.uk/Media/Default/About/ COVID-19/Specialty-guides/specialty-Guide-Virtual-Working-andCoronavirus.pdf

8 Greenhalgh T, Rosen R. Remote by default general practice: must we, should we, dare we? Br J Gen Pract 2021:71:149-50.
9 Curran A, Parle J. Physician associates in general practice: what is their role? Br J Gen Pract 2018;68:310-1.

10 Agarwal R, Mitchell S. Viewpoint: a proposed role for physician associates (PAs) in palliative care. BJGP Life, 02 November 2020 https://bjgplife.com/2020/11/02/viewpoint-a-proposed-role-forphysician-associates-pas-in-palliative-care

11 Agarwal R, Hoskin J. Supervision of physician associates in primary care; the who, what and how is it done? FHJ 2021;8:57-61.

12 Mulvihill C, Cooper ], Pavey ] et al. Remote consultations in primary care during the COVID-19 pandemic: student perspectives. Postgrad Med ] 2020; postgradmedj-2020-139149 [Epub ahead of print].

Address for correspondence: Miss Ria Agarwal, Sothall Medical Centre, Eckington Road, Sheffield S20 1HQ, UK. Email: riaagarwal@nhs.net

Twitter: @riaagarwal19 